\title{
Theorie der Kryptodoxie
}

\section{Erkundungen in den Schattenzonen der Wissensordnung ${ }^{1}$}

\section{Von Michael Schetsche}

Zusammenfassung: Im Anschluss an die Unterscheidung zwischen orthodoxem und heterodoxem Wissen postuliert der Aufsatz die Existenz von innerhalb unserer Wissensordnung gut verborgenem ,Schattenwissen', dessen Gesamtheit mit dem hier neu eingeführten Terminus der Kryptodoxie bezeichnet werden soll. Dieses kryptodoxe Wissen ist monodirektional gegen die geltende Wirklichkeitsordnung abgeschlossen: aus jenen Schattenzonen dringt im Normalfall kein Wissen nach außen. Da diese Abschottung auch jede wissenssoziologische Untersuchung verhindert, kann die Existenz solcher Schattenzonen nur anhand von Beispielfällen belegt werden, bei denen die entsprechenden Wissensbestände eine Zeit lang Teil jener Kryptodoxie waren, dies heute jedoch nicht mehr sind. Im Anschluss an vier, in diesem Sinne notwendig ,historische', Beispiele werden zwei unterschiedliche Prozesse der Entstehung von Schattenzonen und die aus ihnen resultierenden Formen kryptodoxen Wissens vorgestellt: die Tabuisierung und die Ausbildung klandestiner Deutungsmuster. Gemeinsam ist ihnen, dass das kryptodoxe Wissen stets im Modus des reflexiven Geheimnisses vorliegt, das besondere Mechanismen des Wissenstransfers notwendig macht. Solche noch stark theoretisch orientierten Erörterungen verweisen auf ein ausstehendes empirisches Forschungsprogramm zur Wissenssoziologie der Kryptodoxie. Erst dessen Realisierung wird zeigen, wie nachhaltig die Impulse sind, die von der neuen wissenssoziologischen Kategorie für eine tiefgründigere Auslotung unserer Wirklichkeitsordnung - namentlich ihrer Konstituierungsmechanismen und Grenzziehungen - ausgehen können.

\section{Orthodoxie - Heterodoxie - Kryptodoxie}

Wenn wir Berger und Luckmann (1991) folgen, verfügt jede Kultur über eine ihr eigene, mehr oder weniger autochthone ${ }^{2}$ Wissensordnung. $\mathrm{Zu}$ dieser gehören eine oder mehrere (konkurrierende) Weltanschauung(en), eine Vielzahl von Wissensbeständen und Handlungsanleitungen sowie von diesem Wissen hervorgebrachte und kontrollierte Praxisformen der unterschiedlichsten Art. Man kann diese Wissensordnungen in der Nomenklatur von Foucault beschreiben, als System aus Epistemen, Diskursen und Dispositiven, in der Form der klassischen Wissenssoziologie als Sprachen, Ideologien, Deutungsschemata usw. oder - in etwas modernerer Variante - als lebensweltliche Wissenssphäre und diverse Sphären von ,Sonderwissen' (etwa dem religiösen). Den Teil der alltäglichen (aber auch der wissenschaftlichen) Wissensbestände, der sich auf die Beschaffenheit einer als ,objektiv' oder ,wirklich' verstandenen Umwelt $^{3}$ bezieht, kann man als geltendes Wirklichkeitswissen einer Kultur bezeichnen. Eine jeweils konkrete Konfiguration von Wirklichkeitswissen ist dabei nicht typisch für eine bestimmte Kultur, sondern andersherum konstituiert es diese überhaupt erst: Von unterschiedlichen Kulturen sprechen wir immer dann, wenn wir es mit unterscheidbaren Wissensordnungen und von ihnen kontrollierten Wirklichkeitsvorstellungen zu tun haben. Das jeweilige Wirklichkeitswissen sagt uns, in was für einer Welt wir leben, wie wir - als Menschen - darin

1 Der Autor dankt René Gründer, Ronald Hitzler, Ina Schmied-Knittel sowie zwei ihm unbekannten Gutachtern oder Gutachterinnen für eine Vielzahl höchst hilfreicher Anregungen.

$2 \mathrm{Zu}$ fragen wäre in diesem Kontext, ob und in welchem Umfang die Globalisierung als kultureller Prozess die bis vor wenigen Jahrzehnten zu konstatierende autochthone Entstehung einzelgesellschaftlicher Wissensordnungen heute hat obsolet werden lassen. Dies führte an dieser Stelle aber zu weit.

3 Dies ist zu unterscheiden von dem, was man zusammenfassend , fiktionales Wissen' nennen könnte das Wissen über oder von Kulturprodukte(n), die explizit nicht mit dem Anspruch auftreten, die, wirkliche Wirklichkeit' zu beschreiben (etwa Romane, Spielfilme oder Computerspiele). 
unseren Platz finden und auch, welche Handlungsoptionen wir besitzen (und welche eben nicht). Diese Wirklichkeitsordnung ist insofern normativ verbürgt, ${ }^{4}$ als sowohl der geäußerte gedankliche als erst Recht jeder handlungspraktische Verstoß gegen sie entsprechende Sanktionen nach sich ziehen kann; diese reichen von einfacher Stigmatisierung im sozialen Umfeld („der Spinner und sein verrücktes Geschwätz“) bis hin zur Umerziehung oder dauerhaften Aussonderungen von ,Wirklichkeitsrebellen' - in modernen Gesellschaften etwa in psychiatrischen Anstalten (vgl. Foucault 1996: passim).

In komplexeren Gesellschaften enthält die Wissensordnung regelmäßig orthodoxes und heterodoxes Wirklichkeitswissen (vgl. Fuchs-Heinritz / König 2005: 203). Ersteres wird nicht nur von der Mehrheit der Gesellschaftsmitglieder für zutreffend erachtet, sondern auch von den Wissen produzierenden, Wissen verbreitenden und sozialisierend wirkenden Institutionen vermittelt und machtvoll ${ }^{5}$ abgesichert - von der Wissenschaft, den Schulen, den Massenmedien usw. Gleichzeitig zirkuliert in den Kommunikationsmedien (im weitesten Sinne) moderner Gesellschaften immer auch, abweichendes' Wirklichkeitswissen. Es handelt sich dabei um Wissensbestände, die meist nicht die Wirklichkeit insgesamt, aber doch spezifische Teile von ihr anders konstruieren, beschreiben und erklären - und entsprechend auch partiell abweichende Handlungsanleitungen (alternatives Praxiswissen) zur Verfügung stellen. Solche heterodoxen ${ }^{6}$ Wissensbestände gehören nicht immer zum legitimen, aber doch zum bekannten Wissensvorrat der Gesellschaft, befinden sich dabei vielfach in einem gelegentlichen, zyklischen oder auch permanenten, Geltungskrieg' mit der Orthodoxie. Die Rede ist hier von alternativen (vom ,Mainstream' eines Faches nicht anerkannten) wissenschaftlichen Theorien, von abweichenden Alltagsdeutungen (wie etwa den sog. Verschwörungstheorien; vgl. Anton 2011), von mal mehr mal weniger marginalisierten religiösen Interpretationen der Wirklichkeit oder auch von den Überzeugungssystemen bestimmter Subkulturen. Das offene Konkurrenzverhältnis dieses heterodoxen zum orthodoxen Wirklichkeitswissen zeigt sich darin, dass in den orthodoxen Geltungs- und Legitimierungsdiskursen immer wieder (negierend) auf das heterodoxe Wissen Bezug genommen wird und vielleicht auch Bezug genommen werden muss - etwa um es als ideologischen Gegenhorizont zu funktionalisieren. Dabei, und dies ist die Voraussetzung für entsprechende Bezugnahmen, gehören die abweichenden Wirklichkeitsannahmen jedoch zum System des in der betreffenden Kultur Denkbaren (zum geltenden Epistem in Foucaults Sprache): Zwar gilt es aus Sicht der Orthodoxie als höchst unwahrscheinlich, dass jene Wirklichkeitsaussagen zutreffen könnten, gänzlich ausgeschlossen werden kann dies jedoch nicht; es handelt sich sozusagen um Beschreibungen einer potentiellen Wirklichkeit auf Abruf. Konkurrenz besagt hier aber auch, dass nicht ein für alle Mal festgelegt ist, welcher Wissensbestand, welche Theorien und welche lebensweltlichen Deutungen in das orthodoxe und welche in das heterodoxe Segment des Wirklichkeitswissens gehören: Was gestern als

4 Berger / Luckmann (1991: 59) schreiben hierzu: „Die primäre soziale Kontrolle ergibt sich [...] durch die Existenz von Institutionen überhaupt. Wenn ein Bereich menschlicher Tätigkeit institutionalisiert ist, so bedeutet das eo ipso, daß er unter sozialer Kontrolle steht.“.

5 „Macht in der Gesellschaft schließt die Macht ein, über Sozialisationsprozesse zu verfügen, und damit die Macht, Wirklichkeit zu setzen." (Berger / Luckmann 1991: 128).

6 Die zentrale Bestimmung des Begriffs ,Heterodoxie' jenseits der Theologie und Religionswissenschaft, aus der er stammt, findet sich bei Bourdieu (1993: 109): „Diejenigen, die bei gegebenen Kräfteverhältnissen das charakteristische Kapital (mehr oder weniger vollständig) monopolisieren, neigen eher zu Erhaltungsstrategien - Strategien, die im Feld der Produktion kultureller Güter tendenziell die Orthodoxie vertreten -, die weniger Kapitalkräftigen dagegen (die oft auch die Neuen und damit meist Jüngeren sind) eher zu Umsturzstrategien - Strategien der Häresie. Erst die Häresie, die Heterodoxie als kritischer, oft im Zusammenhang mit der Krise auftretender Bruch mit der Doxa, bringt die Herrschenden dazu, ihr Schweigen zu brechen und jenen Diskurs zur Verteidigung der Orthodoxie, des rechten Denkens im doppelten Sinne, zu produzieren, mit dem ein neues Äquivalent zur schweigenden Zustimmung der Doxa geschaffen werden soll.“(Vgl. hierzu auch die Erläuterungen bei Fuchs-Heinritz / König 2005: 201-203). 
wissenschaftlich richtig galt, kann heute theoretisch überholt sein, und was heute als krude Verschwörungstheorie gilt, kann morgen das sichere Wissen über eine tatsächliche Verschwörung darstellen. In diesem Sinne benennt, die Orthodoxie' die Gesamtheit des Wirklichkeitswissens, das in einer bestimmten Kultur gilt, , die Heterodoxie' hingegen die Gesamtheit jenes Wirklichkeitswissens, das dort gelten könnte.

Orthodoxie und Heterodoxie sind in Bezug auf ihre Geltung im Normalfall analytisch gut unterscheidbar, sie stellen allerdings keine kulturell geschiedenen Wissensbestände dar. Anders ist dies bei dem Wissen, dass jenen Bereichen angehört, die ich vorläufig einmal Schattenzonen ${ }^{7}$ der Wissensordnung nennen möchte. Ich behaupte hier (zunächst theoretisch, empirische Studien dazu sollen folgen), dass es metaphorisch gesprochen ,unterhalb', ,neben' oder vielleicht auch ,innerhalb' der verbürgten und eine Kultur ausmachenden Wirklichkeitsordnung gesellschaftlich unsichtbare Zonen des Wissens mit im Allgemeinen gut verborgenen Wissensbeständen gibt. Diese Schattenzonen bestehen - so meine These - aus Wirklichkeitswissen, Handlungsanleitungen und den damit verbundenen Praxisformen, die zumindest teilweise nicht mit der kulturell geltenden Wirklichkeitsordnung kompatibel sind. Entscheidend ist jedoch, dass ihre Wissensbestandteile monodirektional gegen jene geltende Ordnung abgeschottet sind: Aus den Schattenzonen dringt im Normalfall kein Wissen in die geltende orthodoxe Wirklichkeitsordnung (samt ihrer Heterodoxien) hinaus. Letzteres muss dabei als Teil der definitorischen Bestimmung verstanden werden: Aus der Perspektive der herrschenden Wirklichkeitsordnung (Orthodoxie / Heterodoxie) sind jene Schattenzonen unsichtbar.

Man könnte versucht sein, solche Zonen analog zur geltenden Wirklichkeitsordnung als Teil einer fest gefügten Schattenordnung des Wissens zu begreifen. Eine solche Sicht ist jedoch problematisch, weil sich jenes Schattenwissen eben gerade nicht als geschlossene, epistemisch (ab-)gesicherte und gesteuerte Ordnung darstellt - vielmehr sind die einzelnen Wissensbestände hier stark segmentiert und regelmäßig auch gegeneinander abgeschlossen. Wir haben es eher mit einem Konglomerat einzelner, inhaltlich disparater Wissensbestände zu tun, die auf den gesellschaftlich dominierenden Wirklichkeitsparadigmen beruhen können, aber nicht müssen, in jedem Falle jedoch in einem gebrochenen Verhältnis zu diesen stehen, da sie nicht Teil des generellen Prozesses des kulturellen Wissensaustausches sind. Diese sehr disparaten Wissensbestände und Praxisformen können analytisch unter dem Terminus der Schattenzonen zusammengefasst werden, weil sie alle in einem identischen strukturellen Verhältnis zur geltenden Wirklichkeitsordnung stehen. Wenn ich hier im Anschluss an die Unterscheidung von Orthodoxie und Heterodoxie (mit aller Vorsicht) auch von Kryptodoxie spreche, meint dies die Gesamtheit all jenes Wirklichkeitswissens einer Kultur, das aus der Perspektive der herrschenden Wissensordnung so unsichtbar ist, als würde es nicht existieren. Dabei spielt es für dessen Phänomenologie zunächst keine Rolle, ob dieses Wissen kulturell unsichtbar bleibt, weil es nicht diskursivierbar ist, oder ob es nicht diskursfähig ist, weil es unsichtbar bleibt. Dies hängt allein von den Formen ab, die das kryptodoxe Wissen in den strukturell vereinzelten Schattenzonen aufgrund deren jeweiliger Entstehungsbedingungen annimmt.

7 Ich verwende den Begriff ,Schatten' hier im Sinne einer strukturellen Metapher (im Anschluss an Lakoff / Johnson 1980: 14), mit der die grundlegende Asymmetrie der Beziehung zwischen orthodoxem und heterodoxem Wissen auf der einen und kryptodoxem Wissen auf der anderen Seite verdeutlicht werden soll: Ebenso wie Schatten ohne Licht unmöglich ist, bleiben die kryptodoxen Wissensbestände stets auf jenes Wissen (sei es nun anerkannt oder umstritten) bezogen, das gesellschaftlich offen prozessiert wird. Es geht hier also um mehr als nur um die Differenz von individueller Sichtbarkeit und Unsichtbarkeit einzelner Wissensbestände. Insofern unterscheidet meine Begriffsbildung sich sowohl von der Schattenmetapher, wie Berger und Luckmann (1991: 46, 105) sie gelegentlich verwenden, als auch vom kürzlich in der Biologie vorgeschlagenen Konzept einer ,shadow biosphere“ (vgl. Davies et al. 2009), nach dem auf der Erde neben der allgemein bekannten Biosphäre noch eine zweite, bislang unentdeckte ,Sphäre des Lebens' mit anderen biochemischen Basisprozessen existiert. 


\section{Schattenzonen - historische Fallbeispiele}

Zur besseren Nachvollziehbarkeit meiner Ausgangsthese will ich im Folgenden zunächst vier Bereiche $^{8}$ überindividuellen Wissens exemplarisch ansprechen, in denen - mutmaßlich - entsprechende Schattenzonen existieren, richtiger: existiert haben. Bei deren wissenssoziologischer Beschreibung entsteht nämlich eine Paradoxie der Art, wie sie Jean Baudrillard (1978: 16) ähnlich für das Untersuchungsfeld der Ethnologie skizziert hatte:

„Eines Tages im Jahre 1971 hatte die Ethnologie eine flüchtige Begegnung mit ihrem paradoxen Tod. Die Regierung der Philipinen [sic!] beschloß, einige Dutzend Eingeborene der Tasaday, die man im tiefsten Dschungel entdeckt hatte [...] in ihrer totalen Natürlichkeit zu belassen und sie nicht dem Zugriff von Kolonialisten, Touristen und Ethnologen auszusetzen. Damit folgte man einer Initiative von Anthropologen, die ahnten, daß die Tasaday unter ihren Blicken (wie eine Mumie in frischer Luft) förmlich zerfallen würden. "

Wenn Wissensbestände erst einmal wissenschaftlich entdeckt und vermessen sind, können sie nicht mehr oder zumindest nicht mehr gänzlich zu den Schattenzonen der Wissensordnung gehören, wie ich sie verstehe. Dies muss nicht bedeuten, dass sie bereits vollständig Teil der Ordnung der Orthodoxie / Heterodoxie geworden sind, es heißt aber zumindest, dass sie im Hinblick auf jene Wissensordnung nicht mehr im Verborgenen existieren. Die entscheidende Frage lautet dabei, ob jenes Schattenwissen primär in Bezug auf lebensweltliches oder auf wissenschaftliches Wissen definiert wird; gilt Ersteres, können auch wissenschaftlich beschriebene Wissensbestände Teil der Kryptodoxie sein, wenn das entsprechende wissenschaftliche Wissen (noch) nicht in die Lebenswelt transferiert worden ist. In meinen Beispielen kann es gezwungenermaßen nur um Wissensbestände gehen, die bis zu jenem Zeitpunkt zu den Schattenzonen gehörten, als Wissenschaftler, die Massenmedien oder andere Gruppen sozialer Akteure begannen, sie ins Licht zu zerren und ihre Existenz zu verkünden. Wäre dies nicht geschehen, könnte ich meine Beispiele hier nicht entfalten, um mit ihrer Hilfe zu illustrieren, was ich auf der Ebene der Untersuchungsobjekte unter ,Schattenwissen' verstehe. Streng genommen repräsentieren meine Fallbeispiele deshalb kein kryptodoxes, sondern vormals kryptodoxes Wissen - trotzdem, so denke ich, können sie die Existenz der von mir postulierten Schattenzonen in unserer Kultur nachvollziehbar vor Augen führen.

1. Erfahrungen kollektiver Traumatisierungen am Ende des Zweiten Weltkriegs: Im Oktober 2008 berichtete die Online-Ausgabe der ZEIT über den Beginn des ersten wissenschaftlichen Forschungsprojekts zu den Langzeitfolgen der Massenvergewaltigungen bei der Besetzung Deutschlands durch die Alliierten im Jahre 1945. ${ }^{9}$ Obwohl es bereits in den Jahren zuvor vereinzelte Studien zu den damaligen Ereignissen gegeben hatte (Beck 1995; Sander / Johr 2005), dauerte es letztlich mehr als sechzig Jahre, bis es möglich war, zumindest einige der (wenigen noch lebenden) Betroffenen psychologisch auf Spätfolgen ihrer Erfahrungen hin zu untersuchen. Vorher war dies nicht nur für die deutsche Traumapsychologie offenbar ein unmögliches Thema. Mit Recht kann gesagt werden, dass die Massenvergewaltigung deutscher Frauen und Mädchen am Ende des Zweiten Weltkrieges (mit geschätzt 1,4 bis 1,9 Millionen Opfern, so Kuwert et al. 2010: 450) zu den größten Tabus (vgl. Kapitel 3) der Nachkriegsgeschichte gehörte - und zwar in der Bundesrepublik ebenso wie in der DDR. In der DDR durften Berichte über Vergewaltigungen durch Rotarmisten grundsätzlich nicht öffentlich gemacht

8 Meine Fallbeispiele sind schon allein deshalb selektiv, weil sie allesamt aus meinen früheren oder aktuellen Forschungsgebieten stammen. Dass gerade sie am Beginn meiner Auseinandersetzung mit dem Thema ,Schattenzonen der Wissensordnung' stehen, weist darauf hin, dass es einer längeren Beschäftigung mit einem bestimmten Ausschnitt der sozialen Wirklichkeit bedarf, um in diesem das zu entdecken, was es dort eigentlich (also unserer Wirklichkeitsordnung nach) nicht zu sehen gibt.

9 Online-Quelle: http://www.zeit.de/2008/43/studie-kriegsvergewaltigung; Zugriff: 8.12.2010. Erste Ergebnisse der Studien sind kürzlich von Kuwert et al. (2010) veröffentlicht worden. 
werden (vgl. von Münch 2009: 60 f), aber auch in der Bundesrepublik waren die entsprechenden Erfahrungen hunderttausender von Frauen - sei es aus politisch-ideologischen, ${ }^{10}$ sei es aus psychohygienischen Gründen - öffentlich unerwünscht. Es gab, von ganz wenigen Ausnahmen abgesehen, keine wissenschaftlichen Forschungen zu diesem Themenkomplex, die Massenmedien boykottierten das Problem fast vollständig, ein öffentlicher Diskurs fand bis zum Beginn der neunziger Jahre des vergangenen Jahrhunderts nicht statt. ${ }^{11}$ Mindestens ebenso bedeutsam für die Genese einer entsprechenden Schattenzone innerhalb der Wissensordnung war, dass das Thema auch im privaten Bereich nicht angesprochen werden konnte: Während die ,Kriegserlebnisse' der ehemaligen Wehrmachtsoldaten und selbst vieler SS-Männer für Jahrzehnte beliebtes Thema im Freundes- und Kollegenkreis und bei Familienfeiern waren, blieben die Erfahrungen der vergewaltigten Frauen in aller Regel unberichtet (vgl. von Münch 2009: 65). Das entsprechende Wissen wurde höchstens unter den betroffenen Frauen ausgetauscht, vielfach aber nicht einmal an den damaligen oder einen späteren Lebenspartner weitergegeben. Der entscheidende Schritt bei der Ausbildung kryptodoxen Wissens bestand also darin, dass die kollektiven Erfahrungen nicht diskursiv an Dritte (etwa auch die Generation der Nachkriegsgeborenen) weitergegeben wurden. Es blieb bei massenhaften individuellen Erinnerungen, aus denen kein kulturelles Erinnern erwachsen konnte. Dadurch entstand ein erfahrungsbasierter Wissensbereich, der - bis vor wenigen Jahren - kein Bestandteil der herrschenden Wissensordnung sein durfte und sein konnte.

2. Beobachtungen von Wissenschaftlern, die gegen das Wirklichkeitsverständnis ihrer Disziplin verstoßen: Nicht nur in Ethnologie und Anthropologie (sondern auch in anderen Kulturwissenschaften) sorgte Anfang der achtziger Jahre des vergangenen Jahrhunderts eine von Hans Peter Duerr (1981) herausgegebene zweibändige Aufsatzsammlung für einige Aufregung - und manche Irritationen. Unter der Überschrift „Der Wissenschaftler und das Irrationale“ berichteten darin etliche Wissenschaftler, einige davon international höchst renommiert, unter anderem über persönliche Erfahrungen bei (ethnologischen / anthropologischen) Feldforschungen an verschiedensten Orten der Welt, Erfahrungen, die nicht nur im eklatanten Widerspruch zum orthodoxen szientistischen Weltbild des zwanzigsten Jahrhunderts standen, sondern auch - und das ist an dieser Stelle der wichtigere Aspekt - von den Betreffenden vorher entweder vollständig verschwiegen oder höchstens in außerwissenschaftlichem Kontext thematisiert worden waren. Die am Beginn des ersten Bandes (Duerr 1981: 10) gestellte Leitfrage der Anthologie lautete: „Wie verhalten sich Wissenschaftler, wenn sie mit Erfahrungen konfrontiert werden, bei deren Beschreibung oder Erklärung ihre wissenschaftlichen Mittel versagen?" Einer der Autoren, der (damals am zeitlichen Ende seiner wissenschaftlichen Karriere stehende) schwedische Ethnologe Åke Hultkrantz (1981: 74 f), schildert diesen auf den ersten

10 Dies mag damit zusammenhängen, dass erste Thematisierungsversuche in den fünfziger Jahren im Kontext einer politischen, ideologischen und nicht zuletzt wohl auch rassistischen Funktionalisierung stattfanden; so hieß es in einer vom Bundesministerium für Vertriebene, Flüchtlinge und Kriegsgeschädigte herausgegebenen amtlichen Dokumentation zu Flucht und Vertreibung über jene Vergewaltigungen gegen Kriegsende: „Die Tatsache, daß sowjetische Soldaten asiatischer Herkunft sich dabei durch besondere Zügellosigkeit und Wildheit hervortaten, bestätigt, daß gewisse Züge asiatischer Mentalität wesentlich zu jenen Ausschreitungen beigetragen haben“" (zitiert bei Beck 1995: 40). Dies alles ist allerdings nur eine schwache Erklärung dafür, warum das Wissen über jene Massenvergewaltigungen auch in der Bundesrepublik jahrzehntelang ein Schattenthema geblieben ist.

11 Bezeichnend für den hier interessierenden Zusammenhang ist, dass Öffentlichkeit und Wissenschaft in Deutschland sich mit dem Thema ,Massenvergewaltigungen im Krieg' erst angesichts der Ereignisse im Bosnienkrieg (1992-1995) zu beschäftigen begannen (vgl. Beck 1995: 34) - Ereignisse, die offenbar zeitlich wie räumlich weit genug von den Erfahrungen deutscher Frauen am Ende des Zweiten Weltkriegs ,entfernt' lagen, um nicht unter das ursprüngliche Thematisierungsverbot zu fallen. Erst im Reflex auf die Wahrnehmung und Bewertung der Ereignisse in Bosnien setzte in Deutschland eine öffentliche Thematisierung und wissenschaftliche Erforschung der entsprechenden Geschehnisse am Ende des Weltkriegs ein. 
Blick vielleicht verblüffenden Sachverhalt so (ich zitiere hier etwas ausführlicher, um das Gewicht der Aussage deutlich zu machen):

„Als ich mich als angehender Anthropologe daran machte, die Kultur und insbesondere die Religion eines nordamerikanischen Indianerstammes - der Shoshoni in Wyoming - zu untersuchen, hatte ich die Absicht, meine Forschungen durch Interviewtechniken, teilnehmende Beobachtung und Archivauswertungen abzusichern. Ich mußte jedoch feststellen, daß irrationale Faktoren meine sorgfältig ausgetüftelten Vorkehrungen durcheinander brachten, und zwar vor allem auf dem Gebiet der Religion. Aber das war noch nicht alles. An Ritualen mit Medizinmännern teilnehmend, mußte ich in meinem Notizbuch Beobachtungen von einer Art festhalten, die ich in keiner Fachzeitschrift hätte veröffentlichen können, ohne meine wissenschaftliche Reputation aufs Spiel zu setzen! Es ereigneten sich Dinge, die man im Rahmen unseres gängigen wissenschaftlichen Wertesystems nicht als Faktum registrieren konnte. Es war eine eigentümliche Erfahrung, das Verhalten mancher meiner Kollegen in solchen Fällen zu beobachten: wenn sie darüber schrieben oder Vorlesungen hielten, leugneten sie das faktische Vorkommen derartiger Phänomene und führten deren angebliche Existenz entweder auf die Illusion eines verwirrten Geistes oder (was auf dasselbe hinauslief) auf subjektive religiöse Antizipation zurück. Privat äußerten jedoch genau dieselben Kollegen ihre Betroffenheit darüber, derartige Phänomene selbst beobachtet zu haben. Wie ich gestehen muß, war mein eigenes Verhalten nicht viel besser. “

Was Hultkrantz berichtet, ist die Existenz von Schattenwissen innerhalb einer wissenschaftlichen Disziplin (hier der Anthropologie). Es geht um Wissensbestände, die Ergebnis von methodisch einwandfrei durchgeführten Feldforschungen sind, aber niemals auf der , offiziellen Agenda' des betreffenden Faches verhandelt werden konnten. Als Forschungsergebnisse vorgelegt, hätten sie, wenn wir Hultkrantz glauben, den wissenschaftlichen Ruf der Betreffenden zerstört - und zwar ausschließlich aufgrund des spezifischen inhaltlichen Charakters der Beobachtungen bzw. Erfahrungen selbst. Ob diese Schattenzone im Herzen der empirischen Feldforschung heute gänzlich verschwunden ist, kann bezweifelt werden (vgl. etwa Weis 2009). ${ }^{12}$

3. Das Sexualwissen der Kinder: In seinem bis heute einzigartigen Feldforschungsprojekt konnte der Sexualwissenschaftler Ernest Bornemann (1980 a, 1980 b, 1981) zeigen, dass eine den Erwachsenen absolut unbekannte, Welt' geschlechts- und sexualbezogener Wissensbestände (prä-)pubertärer Kinder existiert, die in Form von Liedern, Abzählreimen, Versen, Rätselfragen und ähnlichem tradiert wurden und vielleicht auch heute noch werden. Bornemann interviewte dazu nach eigenem Bekunden zwischen 1960 und 1971 mehr als 2000 Kinder, die er auf großstädtischen Spielplätzen in der Bundesrepublik, der DDR, in Österreich und in der Schweiz ansprach und dazu überredete, ihm solche Reime, Verse usw. vorzutragen, die sie sonst in Anwesenheit Erwachsener eben gerade nicht erzählen würden (vgl. Bornemann 1980 a: 13-32). Solches Wissen war in den sechziger Jahren des letzten Jahrhunderts unter Kindern offenbar weit verbreitet, Erwachsene schienen sich damals jedoch nicht daran zu erinnern, als Kind Entsprechendes gekannt zu haben (Bornemann erklärt dies mit dem von ihm angenommenen Mechanismus der „Pubertätsamnesie“). Über die Tradierungsmechanismen in dieser exklusiven Kinder(wissens)kultur jener Zeit ist bis heute so gut wie nichts bekannt. Die Herkunft jenes Wissens bleibt unklar - auch wenn manche der von Bornemann aufgezeichneten Verse und Lieder Verballhornungen entsprechender Texte der Erwachsenenalltagskultur (von Schlagern über Werbe-Slogans bis zu politischen Parolen) sind.

12 Gegenteiliges signalisierte für die internationale Anthropologie allerdings bereits der Sammelband von Young und Goulet aus dem Jahre 1994; vgl. hierzu auch Marton (2002). 
„Aber die Ursprungsformen sind in eigentümlicher Weise verkindlicht worden, und dieser Prozeß der Verkindlichung besteht nicht etwa darin, daß die Texte vereinfacht oder bereinigt werden, sondern ganz im Gegenteil darin, daß skatologische und sexuelle Aspekte auftauchen, die von Erwachsenen entweder tabuisiert oder, in der erotischen Literatur der Erwachsenen, aufreizend, aufgeilend, verführerisch gestaltet werden." (Bornemann 1980a: 15)

Der Sexualforscher geht davon aus, dass diese ,Texte' wegen der überbordenden sexuellen Anspielungen von den Kindern systematisch vor den Erwachsenen verborgen werden:

\begin{abstract}
„Eines der wenigen Gebiete, wo man, wenn man das Vertrauen des Kindes erwirbt und sich völlig von anderen Erwachsenen distanziert, diese Geheimwelt des Kindes studieren kann, liegt in den Geschichten und Erzählungen, den Anekdoten, Rätseln, Reimen und Versen, die Kinder einander erzählen, wenn sie untereinander sind.[...] Die meisten Eltern wissen nicht, daß es solche Lieder gibt; und da sie es nicht wissen, können sie die Lieder auch nicht verbieten. Trotzdem muß man viele der Lieder als, verboten' bezeichnen, weil sie sowohl im soziologischen wie im psychoanalytischen Sinne tabu sind. " (Bornemann 1980 a: 13-17; Hervorh. von mir)
\end{abstract}

4. Tradierte magische Weltbilder und Praxisformen: Wenn man den öffentlich höchst erfolgreichen Thesen von Doering-Manteuffel (2008) folgt, existierten im Zentrum Europas auch nach der Aufklärung in der Alltagswelt Wissensbestände fort, die die Autorin als „Volksmagie“ und „Wundergläubigkeit" kennzeichnet. Jenseits dieses offen verhandelten und deshalb schon lange zumindest in der Heterodoxie angekommenen Wissens (die Volkskunde untersuchte es das ganze zwanzigste Jahrhundert hindurch unter dem durchaus abwertend gemeinten Schlagwort, Volksaberglaube') existierten bis in die jüngste Vergangenheit hinein noch andere, alles andere als frei zugängliche magiebezogene Wissensbestände. Die Rede ist von einem handlungsorientierten Sonderwissen, das in einzelnen Regionen Europas in eng begrenzten Teilen der Bevölkerung höchst verschwiegen tradiert wurde - möglicherweise über Jahrhunderte. Erst die methodisch neue Wege beschreitenden Untersuchungen von Julio Caro Baroja (1967: 252-266), Jeanne Favret-Saada (1974) und Thomas Hauschild (2002) konnten für einzelne Regionen Spaniens, Frankreichs und Italiens die Existenz dieser klandestinen Wissens- und Praxisformen nachweisen, die lange Zeit auch vor den neugierigen Blicken ,der Wissenschaft' sorgsam verborgen wurden - und es in anderen Teilen Europas vielleicht heute noch werden. ${ }^{13}$ Die genannten Untersuchungen konnten zeigen, dass magisches Denken eben nicht nur als volkstümlich-naive Tradition unter, Unwissenden' fortlebte, sondern bis in die Gegenwart hinein - gleichsam in Nischen der szientistischen Kultur (vgl. Sorell 1991: passim sowie Schmidt-Lux 2008: 31-126) - das grundlegende Wirklichkeitsverständnis kleiner Gruppen von ,Wissenden' prägte und auch (zumindest in den Augen der Beteiligten) höchst wirkungsmächtige okkulte Praktiken anzuleiten vermochte: Fertilitäts- und Heilzauber, Schadensmagie, Divinationspraktiken oder auch verschiedene Formen der Jenseitskommunikation. FavretSaada (1974: 27 f) beschreibt in ihrer Untersuchung die Unmöglichkeit, mit klassischen Methoden der Europäischen Ethnologie (und im Rahmen ihrer herrschenden Paradigmen!) einen Zugang zu den betreffenden Wissensbeständen und Praktiken zu erlangen - und erklärt damit auch, wieso das hier gemeinte Schattenwissen die Bemühungen der traditionellen, Volksaberglaube-Forschung' weitgehend unentdeckt überstehen konnte:

13 Ähnliches für den deutschsprachigen Raum findet sich bei Rudolph (1977), der für seinen Band „Die geheimnisvollen Ärzte“ mehr als 150 traditionelle Heiler („Gesundbeter“) befragte, die überwiegend sehr offen über ihre Fähigkeiten und Praktiken Auskunft gaben - bis auf einige Interviewpartner im norddeutschen Raum, die gewisse, nicht auf Heilvorgänge bezogene magische Praktiken nachdrücklich zu verheimlichen suchten (Rudolph 1977: 101-105). Parallelen zu den aus fast der gleichen Zeit stammenden Berichten von Favret-Saada über das französische Hainland liegen auf der Hand. 
„Als ich mich ins Hainland begab, befand ich mich ganz gewiß in keiner besseren Lage als meine Vorgänger, außer daß mir ihre Funde lächerlich erscheinen im Vergleich zu dem, was bei einem Anfall von Hexerei real auf dem Spiel steht. [...] lachhaft wäre es gewesen, den Versuch zu unternehmen, die Bauern durch großmütige Absichtserklärungen zu verführen, denn wenn es um Zauberei geht, ist es ebenfalls immer der Andere, der die Initiative der Deutung ergreift. [...] Wahrscheinlich war ich noch nicht bereit, diesen Diskurs auf die für meine Gesprächspartner einzig denkbare Weise zu führen: nämlich indem ich anerkannte, daß ich, nachdem man mich auf diesen Posten gestellt hatte, verpflichtet war, einen gewissen Teil dieser Rede persönlich zu halten, so wie es die anderen taten. "In einem Satz: „Der Hexenglaube läßt sich also nicht untersuchen, wenn man nicht bereit ist, in den Situationen, in denen er sich äußert, und in der Rede, die ihn zum Ausdruck bringt, mit eingeschlossen zu werden. "(32)

Es geht mir mithin bei meiner Kryptodoxie-These um alle jene Wissensbestände und die mit ihnen verbundenen Praxisformen, die in einer bestimmten Kultur existieren, aber nicht diskursiviert werden können, also einer öffentlichen Thematisierung (im allerweitesten Sinn des Wortes) dauerhaft entzogen bleiben. Hier stellt sich sofort die Frage, wie es in modernen, von den unterschiedlichsten Formen des Wissenstransfers geprägten Gesellschaften (Stichwort ,Wissensgesellschaft' - mehr dazu im letzten Kapitel) überhaupt möglich ist, dass auf kollektiven Erfahrungen basierende Wissensbestände entstehen und fortexistieren, die vom allgemein zugänglichen Gesamtwissensvorrat der betreffenden Kultur abgetrennt sind. ${ }^{14}$ Wie das Unwahrscheinliche möglich wird, diskutieren die beiden folgenden Kapitel.

\section{Die Entstehung von kryptodoxem Wissen}

Wenn wir im Hinblick auf die vorgestellten Beispiele nach möglichen strukturellen Ursachen für die Entstehung von Schattenzonen der Wissensordnung fragen, ergibt sich das folgende Bild: Das kryptodoxe Wissen in den ersten beiden Beispielen (Massenvergewaltigungen am Ende des Zweiten Weltkriegs; bestimmte ethnologische Beobachtungen) basiert jeweils auf kollektiv-individuellen Erfahrungen mit Widerfahrnis-Charakter. Dieses scheinbare Oxymoron meint Erfahrungen, die zwar von einer großen Zahl von Subjekten in ganz ähnlicher Form gemacht werden, jedoch wegen eines kulturellen Thematisierungsverbots nicht überindividuell ausgetauscht, abgeglichen und bewertet werden können. Es sind dies Erfahrungen, die durchaus auf Basis orthodoxen (erstes Fallbeispiel) oder zumindest heterodoxen (zweites Fallbeispiel $)^{15}$ Wissens gedeutet werden können, aber als Realerfahrungen von den Betroffenen aus kulturellen Gründen nicht kommuniziert werden dürfen. Hier wird deutlich, was einseitiger Abschluss von der Wirklichkeitsordnung meint: Die Erfahrungen wären kulturell prinzipiell verständlich - ein Transfer des konkreten Erfahrungswissens in die Wirklichkeitsordnung ist jedoch unmöglich, weil das betreffende Thema bzw. dessen spezifische Ausprägung tabuisiert ist. Dieses kulturelle Tabu erzeugt eine Schattenzone des Wissens. Der Unterschied zwischen

14 Ohne dass es sich dabei um klassisches, Geheimwissen' handelt, wie es staatliche Instanzen heute in großem Umfang hervorbringen (siehe etwa die massenmedialen Debatten um die Internetplattform Wikileaks, die es sich zur Aufgabe gemacht hat, Teile dieses Wissens öffentlich zu machen). Im Gegensatz dazu geht es mir hier jedoch, um dies noch einmal zu betonen, um Wissen, das prinzipiell als Alltagswissen (oder auch alltagsnahes und erfahrungsbasiertes wissenschaftliches Wissen) angesprochen werden könnte, wenn es denn eben nicht in den Schattenzonen der Wissensordnung verborgen läge.

15 Etwas später in seinem Aufsatz charakterisiert Hultkrantz (1981) die von ihm und seinen Kollegen beobachteten außergewöhnlichen Ereignisse als „,sogenannte(n) Psi-Phänomene(n)“, womit er sie eindeutig als heterodoxes Wirklichkeitswissens rahmt - kryptodox werden die entsprechenden Erfahrungen erst dadurch, dass in der entsprechenden wissenschaftlichen Disziplin jahrzehntelang das von ihm beschriebene Thematisierungsverbot bestand. 
diesen Beispielen (und deshalb führe ich sie hier beide an) besteht darin, dass es sich im ersten Fall um Alltagserlebnisse, im zweiten hingegen um Erfahrungen handelt, die im spezifischen Kontext wissenschaftlicher Forschung gemacht wurden, mithin im Rahmen der methodisch kontrollierten Erzeugung von Wirklichkeitswissen. Dass erfahrungsbasiertes Schattenwissen gleichermaßen in beiden Kontexten entstehen kann, ist ebenso überraschend, wie es weiterer Klärung bedarf.

Beim dritten und vierten Beispiel ist die Entstehungssituation eine andere. Eine Schattenzone konstituierte sich im Fallbeispiel ,kindliches Sexualwissen' deshalb, weil das orthodoxe Wissen zu diesem Feld der betreffenden Personengruppe damals nicht zugänglich war. Damit meine ich, dass Kinder ihren eigenen Körper mit dessen Bedürfnissen sowie bestimmte Geschehnisse der Umwelt wahrnehmen, aber von den zu deren orthodoxer Deutung notwendigen (sexualbezogenen) Wissensbeständen ausgeschlossen werden, weil ihre Kultur dieses Wissen (sei es aus moralischen, pädagogischen oder entwicklungspsychologischen Gründen) systematisch vor Heranwachsenden geheim zu halten versucht. In der Folge entstehen abweichende gruppenspezifische Deutungen von Beobachtungen, die wegen des themenbedingten Ausschlusses von Kindern aus der Diskursgemeinschaft der Erwachsenen dauerhaft nicht mit dem herrschenden Wirklichkeitswissen abgeglichen werden können. So bildet sich eine abgeschottete Sonderzone von sexualbezogenen Deutungen aus, die nicht - wie das entsprechende Sexualwissen der Erwachsenen - auf eigenen Erfahrungen, anerkanntem Alltagswissen und veralltäglichten wissenschaftlichen Modellen beruht, sondern auf einer Mischung von Vermutungen und Ahnungen, irrtümlichen oder auch irreführenden Zuschreibungen und mystifizierenden Deutungen. Aus Sicht der Orthodoxie / Heterodoxie entsteht sexualbezogenes Schattenwissen, das auf eine bestimmte Bevölkerungsgruppe beschränkt bleibt.

Beim Fallbeispiel ,tradierte magische Weltbilder' hat kein Fremd-, sondern in gewisser Weise ein Selbstausschluss von Subjekten aus dem gesellschaftlichen Diskurs stattgefunden. Ursache war wahrscheinlich die Abspaltung bestimmter Segmente überindividuellen Wissens aus der herrschenden Wirklichkeitsordnung: Im Kontext des Prozesses der Aufklärung setzte sich eine rein szientistische Bestimmung von Wirklichkeit durch, in der magische Wissensbestände keinen Raum mehr hatten. Das entsprechende Wissen und die mit ihm verbundenen Praxisformen wurden zunächst (im Kontext der Hexenverfolgung seit Beginn der Neuzeit) normativ-terroristisch und anschließend moralisch-diskursiv marginalisiert und dann gänzlich aus der geltenden Wirklichkeitsordnung ausgeschlossen. Jenes ,magische Weltbild' wurde also im Prozess der Verwissenschaftlichung der Wirklichkeitsordnung zunächst aus der Orthodoxie in die Heterodoxie und dann schließlich von dort in die Kryptodoxie verschoben. Letzteres führte aber wiederum dazu, dass Alltagserfahrungen in bestimmten Bevölkerungsgruppen (namentlich im ländlichen Raum) im Rahmen des nun herrschenden Weltbildes nicht mehr adäquat gedeutet werden konnten - was bezüglich der betreffenden Schattenzone eher als stabilisierendes Element betrachtet werden muss. ${ }^{16}$

Dieser letzte Fall lässt es sinnvoll erscheinen (außerhalb meines eigentlichen Argumentationsganges und deshalb entsprechend kursorisch), nach möglichen theoretischen Verknüpfungen zwischen dem Konzept der Kryptodoxie und aktuellen Debatten einer Soziologie des Vergessens (vgl. Esposito 2002: passim; Connerton 2009: passim; sowie die Beiträge in Dimbath / Wehling 2011 a) zu fragen. Eine erste Antwort ist schnell gegeben: Kryptodoxes Wissen ist nicht individuell , vergessen' und es ist auch nicht kollektiv, vergessen' - und zwar deshalb

16 Interessant ist, dass dieser Prozess auch eine (Teil-)Erklärung für das Verbot der Aufnahme bestimmter Beobachtungen (nämlich jener, die in einen magischen Kontext zu setzen wären) in das wissenschaftliche Wirklichkeitswissen liefert - die Tabuisierung der entsprechenden Erfahrungen von Ethnologen und Anthropologen kann als Sekundärfolge einer Verbannung der diesbezüglichen (magischen) Deutungen aus der Wirklichkeitsordnung der Gesellschaft interpretiert werden. 
nicht, weil diese speziellen, sozial exkludierten Wissensbestände niemals Teil des bekannten und anerkannten Wirklichkeitswissens waren. Vergessen werden im eigentlichen Wortsinne kann nur das, was einmal gewusst wurde (so explizit Dimbath / Wehling 2011 b: 17). Kryptodox im hier gemeinten Sinne ist jedoch das, was - aus unterschiedlichen Gründen - kulturell von vornherein nicht gewusst werden soll oder darf. Lediglich beim letzten Fallbeispiel, den eher kleinräumig tradierten, magischen Weltbildern und Praxisformen, könnten die (wahrscheinlich Jahrhunderte dauernden) Prozesse strategischer Exklusion bei einer weiten Begriffsbestimmung auch als eine Art „,soziales Vergessen“ (Esposito 2002; Dimbath / Wehling 2011 b) interpretiert werden. Ob dies historisch-analytisch sinnvoll ist, müsste ausführlicher diskutiert werden, als es mir hier möglich ist. Stattdessen will ich nur generell festhalten: Die pauschale Anwendung von Überlegungen zum sozialen Vergessen auf meinen Gegenstand scheint mir eher problematisch, weil Schattenzonen des Wissens, wie oben (Kapitel 1) bereits ausgeführt, eben keine epistemisch geschlossene Schattenordnung bilden, vielmehr voneinander getrennt existieren und vermutlich in ihrer Entwicklung auch einer jeweils spezifischen Eigenlogik folgen. Aus diesem Grunde müsste in jedem Einzelfall geprüft werden, welche Bedeutung Prozesse des Erinnerns und Vergessens jeweils (gehabt) haben. Relevant wäre die Frage nach der Möglichkeit des Erinnerns sicherlich immer dort, wo es darum geht, wie kryptodoxes Wissen unter prekären sozialen Transferbedingungen überhaupt bewahrt werden kann (mehr dazu im 4. Kapitel). Insofern scheinen mir Probleme des kollektiven (wenn eben auch gerade nicht kulturellen!) Erinnerns hier letztlich bedeutsamer als jene des Vergessens (was ganz unstrategisch, gleichsam automatisch geschieht, wenn der intergenerationelle Wissenstransfer versagt). Zukünftig könnte im Anschluss an die aktuellen Debatten zur Dialektik von kollektivem Erinnern und Vergessen allerdings durchaus gefragt werden, welche empirischen oder theoretischen Befunde hier auf die Ausbildung einzelner Schattenzonen des Wissens übertragen werden könnten. Ob die Formel, Vergessen durch Exklusion' bzw. ,Exklusion als Vergessen' in Einzelfällen tatsächlich Sinn macht, muss empirisch entschieden werden.

Um zu meiner Argumentationslinie zurückzukehren: Die strukturellen Ursachen der Entstehung von Schattenwissen sind [a] die Tabuisierung bestimmter kollektiv-individueller Erfahrungen, die dann aufgrund des bestehenden Kommunikationsverbots für die Subjekte besonderen Widerfahrnis-Charakter bekommen, [b] der systematische Ausschluss von Subjekten - im dritten Fallbeispiel: einer bestimmten Altersstufe - von der Teilhabe an spezifischen Segmenten des herrschenden Wirklichkeitswissens (was strukturelle Deutungsdefizite zur Folge hat, die durch die Ausbildung aparter Deutungsmuster ausgeglichen werden müssen), [c] die kulturelle (Ver-)Bannung bestimmter historisch vorhandener Segmente von Wirklichkeitswissen aufgrund der Änderung der dominierenden Paradigmen bzw. Episteme. Ob im Falle anderer Schattenzonen der Wissensordnung jeweils einer dieser Prozesse eine Mischung von diesen oder noch gänzlich andere Faktoren zur Entstehung kryptodoxen Wissens führte, wäre zukünftig im Rahmen fallanalytischer Rekonstruktionen durch die empirische Wissenssoziologie (wie Berger und Luckmann - 1991: $15 \mathrm{f}$ - sie eingefordert hatten) zu untersuchen.

Auf Basis der vorgestellten Fallbeispiele kann immerhin schon einmal gesagt werden, dass kryptodoxes Wissen uns in mindestens zwei unterschiedlichen Formen gegenübertreten kann: Als Tabuisierung / Tabu ${ }^{17}$ einerseits und als ein abweichendes klandestines Deutungsmuster andererseits. Diese beiden unterschiedlichen Formen stehen in den hier diskutierten Fällen in direktem strukturellem Zusammenhang mit dem Prozess der Entstehung der betreffenden Schattenzone. Jene Prozesse ermöglichen es, dass Wissensbestände , wissenstechnisch' vom allgemein zugänglichen Wissenskorpus der Gesellschaft abgetrennt und in Schattenzonen der Wissensordnung verschoben werden. Diese Prozesse und die von ihnen konstituierten Wissensformen sind deshalb gleichzeitig auch als Mechanismen zu verstehen, die verhindern, dass

17 An dieser Stelle müssen Prozess und Struktur als Einheit begriffen werden. 
es zu einem späteren Zeitpunkt zum Transfer von Wissen aus jenen Schattenzonen in die herrschende Wissensordnung kommt, was die schnelle Auflösung der entsprechenden Zonen zur Folge haben müsste. (In welchen Kontexten und mit welchen Folgen solche Absicherungsmechanismen dann eben doch ,versagen', ist für die vorgestellten Beispiele in Kapitel 5 skizziert.) Wie monokausal und monodirektional dieser Zusammenhang regelmäßig sein wird und welche anderen Formen kryptodoxen Wissens noch vorfindbar sind, würde wohl erst eine Komparatistik auf Basis einer größeren Zahl von Fallstudien aufzeigen können. An dieser Stelle muss ich mich damit begnügen, kurz die beiden - durch unterschiedliche Entstehungsprozesse bedingten - Formen kryptodoxen Wissens vorzustellen, die bereits bekannt sind:

Form I: Das kulturelle Tabu: Der Begriff Tabu stammt aus dem polynesischen Sprachraum und hat über Reiseberichte und die frühe Ethnologie Eingang in die deutsche Sprache gefunden. Während der Terminus ursprünglich eine deutlich religiöse Konnotation (die entsprechenden Gegenstände ${ }^{18}$ gelten entweder als heilig und deshalb unberührbar oder aber als unrein bzw. gefährlich und deshalb zu meiden - manchmal auch beides in einem) besaß, wurde er im Deutschen schnell säkularisiert und benennt heute ein Handlungs-, Rede- oder sogar Denkverbot hoher Intensität. Ein Tabu ist kurz gesagt das, über das unter keinen Umständen gesprochen werden darf... und über das das Subjekt manchmal auch aus psychischen Gründen gar nicht sprechen kann. Ersteres wird normativ garantiert (mit entsprechenden Sanktionen für Tabu-Verletzer), Letzteres sozialisatorisch, indem psychische Instanzen im Subjekt geschaffen werden, die es daran hindern, bestimmte Dinge zu tun, bestimmte Themen anzusprechen oder auch nur in Ruhe über sie nachzudenken. Bezüglich der innerpsychischen, aber eben auch der sozialpsychologischen Mechanismen sind bis heute die von Freud (insbesondere im Werk „Totem und Tabu“ von 1913) vorgenommenen definitorischen Setzungen von besonderer Bedeutung. Folgen wir dem, wirkt das kulturelle Tabu im Gegensatz zur expliziten Norm stärker ,automatisiert' und auch die Sanktionen, die auf die Tabuverletzung folgen, bedürfen keiner menschlichen Akteure, die sie exekutieren:

„Die Strafe für die Übertretung eines Tabu wird wohl ursprünglich einer inneren, automatisch wirkenden Einrichtung überlassen. Das verletzte Tabu rächt sich selbst. Wenn Vorstellungen von Göttern und Dämonen hinzukommen, mit denen das Tabu in Beziehung tritt, so wird von der Macht der Gottheit eine automatische Bestrafung erwartet. In anderen Fällen, wahrscheinlich infolge einer weiteren Entwicklung des Begriffs, übernimmt die Gesellschaft die Bestrafung des Verwegenen, dessen Vorgehen seine Genossen in Gefahr gebracht hat. " (Freud 2007: 68)

Sei es in traditionalen oder in modernen Gesellschaften, durch die Setzung eines Tabus wird der Austausch von Wissen zu den betreffenden Topoi erst einmal unterbunden. Der Generation, die nach der Setzung des Tabus in die Welt tritt, wird nur noch signalisiert: hierüber sprechen wir nicht; später fällt möglicherweise sogar die Möglichkeit fort, das Tabu auch nur zu benennen, weil die entsprechenden Begrifflichkeiten selbst nun der Tabuisierung zum Opfer gefallen sind. Ein schönes historisches Beispiel hierfür stellt die Entwicklung des kulturellen Umgangs mit der kindlichen Onanie in der Moderne dar. Am Ende jenes Prozesses der Tabuisierung steht ,die (scheinbare) Verbannung der gefährlichen und triebhaften Sexualität aus dem Bewusstsein der Individuen. Im Onaniediskurs ist diese Schwelle in der Mitte des 19. Jahrhunderts erreicht, als man dazu übergeht, die Handlungen selbst nicht mehr beim Namen zu nennen, sondern verhüllend zu umschreiben. Das Kind soll nur noch ahnen, worum es geht" (Schmidt / Schetsche 2009: 46). Generell gilt: Solche Tabus schützen ein Thema vor der Diskursivierung und damit auch vor der Weitergabe und Weiterentwicklung der entsprechenden

18 „Als Quelle des Tabus wird eine eigentümliche Zauberkraft angesehen, die an Personen und Geistern haftet und von ihnen aus durch unbelebte Gegenstände hindurch übertragen werden kann“, heißt es dazu bei Freud (2007: 68). 
Wissensbestände außerhalb eng definierter Gruppen von ,Wissenden' - entsprechend können sie Schattenzonen in der Wissensordnung erzeugen und auch dauerhaft bewahren.

Form II: Das abweichend-klandestine Deutungsmuster: In ihrer wissenssoziologisch konturierten Deutungsmustertheorie bestimmten Plaß und Schetsche (2001) soziale Deutungsmuster als spezifisch strukturierte Wissensbeständen, die in unserer Gesellschaft überall da auftreten, wo kollektives Wissen Situationsdeutungen und aus diesen resultierende Handlungsanleitungen generieren muss. ${ }^{19}$ Diese Deutungsmuster (seien es orthodoxe oder auch heterodoxe) werden medial verbreitet und erlangen ihr Geltung bzw. - im Fall der Heterodoxie - die Möglichkeit einer zukünftigen Geltung eben über jenen Austausch unter den Subjekten, die zugleich Träger und alltägliche Anwender der entsprechenden Deutungsmuster sind.

„, Solange uns eine Situation ein-deutig erscheint (das heißt: sich zweifelsfrei unter ein Deutungsmuster subsumieren läßt), werden wir so handeln, wie es uns aufgrund der Deutung als allein richtig erscheint. Wir können Deutungsmuster deshalb auch als fraglose Normen auffassen, die unsere Interaktionen regeln. Die Sanktionen dieser impliziten Normen sind das Nichtverstehen und das Nicht-Verstanden-Werden. Regelmäßige Abweichungen von der Norm führen zu faktischem Ausschluß aus der Gemeinschaft [...]. " (Schmidt I Schetsche 1998: 24)

Eine andere Art des Ausschlusses aus der Gemeinschaft, die die oben genannten sozialen Funktionen von Deutungsmustern zumindest partiell zusammenbrechen lässt, können wir bei jenen Deutungen beobachten, die ich als , abweichend-klandestin' bezeichnen will. Sie entstehen entweder (wie im dritten Fallbeispiel) aufgrund des Ausschlusses einer bestimmten Gruppe von Subjekten aus jenem gesellschaftlichen Diskurs, in dem die entsprechenden Deutungsmuster verbreitet und aktualisiert werden, oder (wie im vierten Fallbeispiel) durch die diskursive - und auch normativ-handlungspraktische - Absonderung bestimmter Deutungsmuster eben aus jenem Diskurs. In dem einen Fall entstehen unter den so ausgeschlossenen Personen alternative Deutungen bestimmter Lebenssachverhalte und sozialer Situationen, die jedoch innerhalb dieser neuen exkludierten ,Sonderdiskursgemeinschaft' kommunikativ eingeschlossen bleiben (müssen), weil die Betreffenden sich nicht am gesellschaftlichen Diskurs beteiligen dürfen. Foucault (1991: 5-30) rekonstruierte dies als jene Prozeduren, die über die Bestimmung von Sprecherrechten Diskurse zu kontrollieren vermögen. In dem anderen Falle halten sich die traditionellen, nun aber, verbotenen' Deutungsmuster in bestimmten Teilen der Bevölkerung, was diese in Bezug auf jene Deutungen wiederum zu einem (thematisch begrenzten) Selbstausschluss aus der allgemeinen Diskursgemeinschaft zwingt. Das Ergebnis ist in beiden Fällen jedoch das gleiche: Nun existieren bestimmte Deutungen der Wirklichkeit, die auf einen begrenzten Personenkreis beschränkt sind und auch - aufgrund des normativen Drucks der Gesamtkultur - beschränkt bleiben müssen. Diese Deutungsmuster weichen ebenso von den anerkannten (oder zumindest als alternativ diskutierten) Wirklichkeitskonstrukten ab, wie sie klandestin sind und bleiben - solange sie im Verborgenen verbreitet und angewendet werden. Dies verweist auf den entscheidenden Mechanismus der Aufrechterhaltung solcher Schattenzonen: die Geheimhaltung.

\section{Geheimnisse und ihre Tradierung}

Aus Sicht der Orthodoxie / Heterodoxie liegt kryptodoxes Wissen regelmäßig in Form von Geheimnissen vor - und wird auch so prozessiert. Eine genauere Bestimmung dieser Wis-

19 Neben der überindividuellen Steuerung von Handlungen und Interaktion erfüllen Deutungsmuster vier zusätzliche Funktionen für Subjekt und Gesellschaft: Komplexitätsreduktion, Antizipation von Situationsentwicklungen, Verständigung über Grenzsituationen und Erzeugung sozialer Gemeinschaft (vgl. Plaß / Schetsche 2001: 522-527). 
sensform könnte entsprechend in Anschluss an theoretische Überlegungen (etwa bei Simmel 1907, 1958; Sievers 1974; Westerbarkey 1991; Bellebaum 1992) erfolgen. An dieser Stelle muss eine einfache Bestimmung genügen: Aus wissenssoziologischer Warte „bezeichnet ,das Geheimnis‘ eine asymmetrische soziale Beziehung bezüglich der sozialen Ressource Wissen. Das Geheimnis benennt dabei ebenso ein spezifisches Verhältnis von Subjekten oder Gruppen zu Informationen, wie es dieses Verhältnis durch seine Existenz überhaupt erst herstellt“ (Schetsche 2008: 37 f). Seine soziale Bedeutung erhält das Geheimnis über Prozesse sozialer Inklusion und Exklusion, indem es in einer bestehenden Kultur Wissende von Unwissenden trennt. ${ }^{20}$ Mit dem Geheimnis kann soziale (politische, wirtschaftliche) Macht verbunden sein, wenn es seinen Trägern Schlüsselstellungen in den entsprechenden Subsystemen der Gesellschaft verschafft oder ihnen hilft, diese zu sichern. Das Geheimnis kann aber auch zur Überlebensnotwendigkeit werden, wenn das betreffende Wissen, das im Geheimnis kodiert ist und durch dieses geschützt wird, sozial hoch stigmatisiert ist und seine Träger kulturellen oder auch politischen Sanktionen ausgesetzt wären, würden sie sich zu diesem Wissen bekennen. Letzteres ist bei dem hier interessierenden kryptodoxen Wissen regelmäßig der Fall. Aus diesem Grund dürfte Schattenwissen primär in jener Sonderform vorliegen, die Sievers (1974) ,reflexives“, Goffman (1991: 129) hingegen „Zweifaches“ oder „dunkles“ Geheimnis genannt hat. Die reflexive Geheimhaltung spendet „Schutz und Sicherheit, sie befreit den Geheimhaltenden vor den Reaktionen des Ausgeschlossenen bezüglich dessen Ausgeschlossenseins; Rechtfertigungen und Erklärungen des Geheimnisbesitzes werden überflüssig, die Gefahr des Herausforschens des von ihm verborgenen Inhalts hat er - zumindest unmittelbar - nicht zu fürchten" (Schirrmeister 2004: 58). Dies ist im Falle der Schattenzonen entscheidend, weil bereits das Wissen über die Existenz der betreffenden Wissensbestände diese aus der Sphäre der Kryptodoxie in die Ordnung der Orthodoxie / Heterodoxie überführen könnte: Die Wissensbestände, obwohl ihrem Inhalt nach unbekannt, wären im Diskurs als solche adressierbar und würden damit auch Teil eben dieses allgemeinen Diskurses (wenn auch vielleicht zunächst des heterodoxen). Dies wäre der erste Schritt zur Destabilisierung der entsprechenden Schattenzone der Wissensordnung.

Wenn das betreffende Wissen (das erste meiner Beispiele oben suggeriert, dass es entsprechende Fälle geben könnte) nicht ohnehin dazu bestimmt ist, von seinen Trägern bzw. Trägerinnen mit ins Grab genommen zu werden, entsteht bei reflexiver Geheimhaltung das Problem, wie das Geheimnis weitergegeben werden kann, ohne dass seine Existenz oder gar sein Inhalt offenbar werden. Es müssen also Mechanismen des Wissenstransfers existieren, die einerseits aus Sicht der Orthodoxie / Heterodoxie als solche unsichtbar bleiben, andererseits aber sicherstellen, dass das kryptodoxe Wissen die nächste Generation ihrer Träger möglichst vollständig und unverändert erreicht. Der einfachste Transfermechanismus ist hier jener der direkten personale Weitergabe von Wissen unter Ausschluss aller medialen Vermittlung - also von Wissendem (alt) zu Wissendem (neu), wie wir es auch bei der Weitergaben von esoterischem Geheimniswissen finden, etwa als individuelle Vermittlung von einem Lehrer an einen ausgewählten Schüler.

Deutlich komplizierter wird die Sache, wenn aufgrund der äußeren Umstände (etwa der strukturellen Organisation der betreffenden Schattenzone) eine direkte personale Weitergabe von Wissen nicht stattfinden kann. Dann müssen Medien eingesetzt werden, und zwar auf eine Weise, die sicherstellt, dass nicht mitgemeinte Dritte keinen Zugang zu dem entsprechenden Wissen erhalten. Die benutzen Transfermedien könnten (wie etwa manche Texte gnostischer

20 Die für das vollständige Verständnis des Geheimnisses als Wissensform notwendige triadische Bestimmung (das unbekannte, das bekannte und das gewusste Geheimnis) mit den entsprechenden drei primären Rollen (des Wissenden, des Mitwissers und des Unwissenden) kann für die hier interessierenden Zusammenhänge zur dualen Form vereinfacht werden (vgl. Schetsche 2008: 38). 
Gruppierungen in den ersten Jahrhunderten unserer Zeitrechnung) an schwer zugänglichen und geheim gehaltenen Orten versteckt werden; in diesem Falle muss lediglich die Information bewahrt werden, wo die entsprechenden Dokumente sich befinden, um eine Tradierung über Generationen hinweg zu realisieren. Eine zweite Möglichkeit ist die Verschlüsselung von Wissen, so dass es für ,Uneingeweihte' nicht zu rezipieren ist. Schon die Offenbarung der Existenz von solch verschlüsseltem Wissen kann allerdings, wenn denn der Kontext zu rekonstruieren ist, in dem es entstand, riskant für die Existenz der entsprechenden Schattenzone sein: Die verschlüsselten Dokumente werden (wie etwa das Voynich-Manuskript aus dem 15. Jahrhundert) ${ }^{21}$ nach ihrem Auffinden schnell Teil erst eines heterodoxen und dann vielleicht sogar des orthodoxen Diskurses. Besser gefeit gegen ein solches ,Zusammenschmelzen' wäre Schattenwissen durch eine Verschlüsselung zweiten Grades, bei dem der unwissende Betrachter (eines Textes, eines Bildes, eines Filmes usw.) nicht einmal bemerkt, dass neben der offensichtlichen noch eine versteckte Botschaft existiert. (Den hiermit verbundenen wissenssoziologischen und bildwissenschaftlichen Fragen zu folgen würde an dieser Stelle zu weit führen.)

Entscheidend ist in jedem Fall, dass kryptodoxe Wissensbestände nicht offen tradiert und damit auch nicht auf die sonst in der Gesellschaft üblichen Weise transferiert werden können. Sie müssen personal und ,im Geheimen' weitergegeben oder in einer Art und Weise verborgen bzw. verschlüsselt werden, die ihre Herkunft und ihren Entstehungskontext verschleiert - aber trotzdem einen Zugriff jener Personen möglich macht, die Träger des kryptodoxen Wissens werden sollen. Nur wenn dies gelingt, kann Schattenwissen über die Generationen ihrer ursprünglichen Träger hinaus weiterexistieren. Im anderen Falle wird das entsprechende Wissen entweder Teil der Orthodoxie / Heterodoxie - oder es entschwindet ganz einfach aus der Geschichte, als hätte es nie existiert. Hier wäre weitergehend zu fragen, ob die, rückstandslose kulturelle Eliminierbarkeit' generell ein konstitutives Element kryptodoxen Wissens ist - ein Risiko, von dem heterodoxes Wissen wegen seiner argumentativen wie organisatorischen Kopplung an die Orthodoxie nicht in gleicher Weise betroffen ist.

\section{Wissenssoziologie der Kryptodoxie}

Die von der Theorie der Kryptodoxie postulierte Existenz des Unsichtbaren mitten im Sichtbaren verweist zunächst auf die Unvollständigkeit unseres soziologischen Bildes vom Wissen moderner Gesellschaften. Das Besondere an Schattenzonen der Wissensordnung ist, dass sie einseitig gegen das gemeinsam von Orthodoxie und Heterodoxie gebildete Wirklichkeitswissen abgeschottet sind. Wissensbestände aus der geltenden Wirklichkeitsordnung können die Grundlage auch des Schattenwissens sein (wie das lebensweltliche Wissen die Basis aller Formen von Sonderwissen ist), müssen dies - wie insbesondere das Beispiel des kindlichen Sexualwissens zeigte - aber nicht notwendig. Entscheidend ist vielmehr, dass es für Wissen aus den Schattenzonen im Normalfall keinen, Weg zurück' in die herrschende Wissensordnung gibt. Weil das Wissen der Schattenzonen per Definition im Verborgenen gedeiht und um den Preis seiner Existenz strukturell gegen seine Offenbarung und Verbreitung abgeschottet sein muss, kann kein Wissenstransfer von hier aus zur , sichtbaren Seite' der Wissensordnung stattfinden. Über jenes Schattenwissen ist deshalb aus Sicht des orthodoxen Wissenssystems (und namentlich der Wissenschaft) nur zufällig oder über außergewöhnliche Anstrengungen etwas zu erfahren. Dies geschieht meist, wenn die entsprechende Schattenzone sich bereits in Auf-

21 Ein wahrscheinlich aus Italien stammendes Manuskript, das (neben zahlreichen verwirrenden Zeichnungen) große Textmengen in unbekannter Schrift und Sprache enthält. Bis heute ist umstritten, ob es sich um ein ernst zu nehmendes okkultes Manuskript mit verschlüsseltem Geheimwissen der Frührenaissance oder um eine reine Phantasiekreation, etwa aus künstlerischer Intention, handelt. (Vgl. Roitzsch 2008). 
lösung befindet - falls es nicht sogar die entsprechenden Forschungsaktivitäten selbst sind, welche zur Dekomposition führen. Die Beispiele des zweiten Kapitels sollten dies deutlich gemacht haben.

Das Schattenwissen über die Massenvergewaltigungen am Ende des Zweiten Weltkriegs schwand (wie bereits erwähnt) im Kontext der öffentlichen Debatten über ähnliche Verbrechen im Bosnien-Krieg dahin. Hierzu trugen insbesondere ein Dokumentarfilm aus dem Jahre 1992 („BeFreier und Befreite“ von Helke Sander) und die 2003 veröffentlichten anonymen Tagebuchaufzeichnungen aus den letzten Kriegstagen (Enzensberger 2003) ${ }^{22}$ bei. Das sexuelle Wissen der Kinder versuchte uns ein solitär arbeitender Sexualwissenschaftler mit seinem dreibändigen Feldforschungsbericht (Bornemann 1980 a, 1980 b, 1981) nahezubringen, ein Bericht, dessen Befunde bis heute allerdings weitgehend unrezipiert geblieben sind. Die höchst okkulten magischen Praxen in manchen europäischen Ländern in der zweiten Hälfte des 20. Jahrhunderts wurden hingegen (mehr oder weniger erfolgreich) tatsächlich durch jene europäischen Ethnologen ,enttarnt', die sich in mehrfacher Hinsicht nicht mit dem zufriedengeben wollten, was die Volksaberglaube-Forschung in den Jahrzehnten zuvor schon ans Licht gezerrt hatte. Und das Schattenwissen innerhalb ihrer Disziplinen selbst offenbarten Anthropologen und Ethnologen, die sich - meist ganz am lebenszeitlichen Ende ihrer wissenschaftlichen Karriere stehend - nicht mehr mit der wissenschaftlichen Unveröffentlichbarkeit bestimmter empirischer (Be-)Funde abfinden wollten. ${ }^{23}$

Zusammenfassend kann man auf Basis der (wenigen) Beispiele festhalten: In einigen Fällen hat die themenbezogene wissenschaftliche Forschung die Existenz der betreffenden Schattenzonen offenbart, in anderen Fällen waren die Träger des Wissens selbst oder mit ihnen verbündete Akteure (etwa Experten oder Massenmedien) am Werk. In jedem Fall aber haben die entsprechenden ,Offenbarungen' die betreffende Schattenzone aufgelöst oder doch zumindest der Auflösung nahe gebracht. Für die wissenschaftliche Erforschung der Kryptodoxie bedeutet dies, dass die Wissenssoziologie entsprechende Schattenzonen wahrscheinlich immer erst dann wird rekonstruieren können, wenn deren Ende naht oder schon besiegelt ist. Im Gegensatz zum eingangs zitierten Vorwurf Baudrillards an die Adresse der Ethnologie (für den ,Tod' jener Stämme verantwortlich zu sein, die sie zu ihrem Untersuchungsobjekt gemacht haben), wird es dabei aber eher nicht die Wissenssoziologie selbst sein, die die entsprechende Schattenzone de-konstruiert - diese Aufgabe werden zumeist die für den Wissensbereich jeweils zuständigen Fachdisziplinen übernehmen (in den genannten Beispielen etwa die Sexualwissenschaft oder die Ethnologie). Dem Wissenssoziologen wird lediglich, um einmal eine drastische Metapher zu benutzen, die Aufgabe des Pathologen zukommen, der nach der Sektion zu berichten hat, wer das Opfer war, wie es ihm zu Lebzeiten ergangen ist - und wie es dann mutmaßlich zu Tode kam. Ob es darüber hinaus auch möglich ist, auf Basis der vorgestellten theoretischen Überlegungen die Existenz von Schattenzonen an ganz bestimmten, Orten' der Wissensordnung zu prognostizieren, ohne dass das konkrete Schattenwissen empirisch offenbart würde, wird sich zeigen müssen.

Abschließend ist vielleicht noch zu fragen, welche Bedeutung der generellen Pluralisierung von Wissen in der modernen Gesellschaft für die Entstehung, Persistenz und die Auflösung kryptodoxer Wissensbestände zukommen könnte. Bereits Berger und Luckmann (1991) sprachen ja von einer „Hierarchie der Wirklichkeiten“ (105) und schilderten an anderer Stelle (121-124) ausführlich, was mit Anhängern abweichenden Wirklichkeitswissens geschieht.

22 Eine größere Öffentlichkeit erreichte die Verfilmung aus dem Jahre 2008 („Anonyma - Eine Frau in Berlin“, Regie: Max Färberböck).

23 Ob diese plötzlich auftretende, Offenbarungsbereitschaft' eher biographische Gründe hatte oder auch mit Veränderungen im Selbstverständnis der betreffenden Disziplinen einherging (Stichwort: Postkolonialismus-Debatte), wäre noch zu prüfen. 
Besondere Bedeutung schrieben sie Konkurrenzprozessen zwischen alternativen Wirklichkeiten in modernen Gesellschaften westlichen Typs (die Autoren hatten hier die USA vor Augen) zu: „In einer Gesellschaft, die konträre Welten öffentlich auf dem Markt feilbietet, werden für subjektive Wirklichkeit und Identität gewisse Konsequenzen gezogen. Das allgemeine Gefühl für die Relativität aller Welten nimmt zu - einschließlich der eigenen, die subjektiv als eine Welt, nicht als die Welt angesehen wird“ (184). Die seither immer wieder diskutierte Pluralisierung von Wissen in eben jener so genannten Wissensgesellschaft (vgl. etwa Degele 2000: 261-292; Böhle 2003; Knoblauch 2005: 267-284; Pscheida 2010: 214-221) betrifft nach meiner Wahrnehmung primär die Konkurrenz zwischen jenen - mal mehr, mal weniger offen verhandelten - Wirklichkeitsdeutungen, von denen bereits bei Berger und Luckmann die Rede war. Besonders schwergewichtig erscheint mir die Pluralisierungsthese aktuell im Kontext der von den Netzwerkmedien hervorgerufenen technisch-kulturellen Veränderungen: Die Frage, was in einer Gesellschaft als wirklich gilt, ist im ,Zeitalter' der Netzwerkmedien möglicherweise nicht mehr so leicht zu beantworten, wie in jenem der Massenmedien. Die von Luhmann (1996: 9-23) für Letztere postulierte Realitätssetzungsfunktion ${ }^{24}$ scheint von Ersteren nicht oder zumindest nicht in gleichem Maße erfüllbar. In lebensweltlichen wie wissenschaftlichen Debatten wird angenommen, dass die Netzwerkmedien eher individuelle ,Lesarten' von Wirklichkeit fördern (oder gar produzieren), statt eine einheitliche Wirklichkeitsordnung zu konstituieren (vgl. Eid 2002; Pscheida 2010: 240-244 und 415-429). Doch selbst wenn diese These zutreffen sollte (die Rolle zumindest der aktuell dominierenden netzwerkmedialen Einstiegsportale, Google und Wikipedia, kann auch ganz anders verstanden werden ${ }^{25}$ ), bedeutet dies für den hier diskutierten Kontext zunächst einmal nur, dass die traditionelle Trennung zwischen Orthodoxie und Heterodoxie unschärfer und durchlässiger wird, sich hier und da vielleicht sogar auflöst. Ob dies auch für die nach meinem Verständnis deutlich schärfere Scheidelinie zwischen der Wissensordnung und ihren verschiedenen Schattenzonen gilt, ist hingegen eine ganz andere Frage.

Verdeutlichen lässt sich dies an dem oben verwendeten Beispiel des kindlichen Sexualwissens. Da die vorgestellte Untersuchung von Bornemann nur wenig rezipiert wurde und seine Befunde bis heute kaum Eingang in das anerkannte (sexual-)pädagogische Wissen gefunden haben, scheinen wir es hier auf den ersten Blick mit einen Fall zu tun zu haben, in dem die entsprechende Schattenzone inzwischen wieder weitgehend intakt ist. Gegen diese Vermutung könnte die aktuelle Debatte über die ,sexuelle Verwahrlosung der Jugend' (vgl. die Beiträge in Schetsche / Schmidt 2010) sprechen. In jenem Problemdiskurs geht es primär allerdings um (oftmals schon sexuell aktive) Jugendliche und nicht, wie in der Studie von Bornemann, um präpubertäre Kinder. Trotzdem ist zu fragen, ob nicht die in diesem Kontext diskutierte Aufhebung der Trennung zwischen den Sphären ,öffentlich und privat' durch die Nutzung der Netzwerkmedien (vgl. Schetsche 2010: 330-335) die Existenz jener „Geheimwelt des Kindes“ gefährdet, von der Bornemann zu seiner Zeit noch sprechen konnte. Dieser Verdacht liegt deshalb nahe, weil es gerade die jüngeren und jüngsten Nutzer sind, die in jenem Medium neue Verhaltens- und Moralstandards setzen (vgl. Klein 2010; Richard 2010). Alternativ wäre allerdings auch vorstellbar, dass jene ,Welt der Netze' gerade für diese jungen Nutzer neue Kommunikationswege bereitstellt, welche die Abtrennung und Geheimhaltung der Wissensbestände - seien sie sexualbezogen oder nicht - einer originären ,Kinderwirklichkeit' dauerhaft sogar erleichtert. Möglich ist schließlich sogar, dass der Einfluss medialer Kommunikation auf die Denkwelt präpubertärer Kinder im aktuellen Diskurs überschätzt wird und eine Studie wie die von Bornemann heute eben doch zu ganz ähnlichen Ergebnissen führen würde.

24 Vgl. dazu aus sozialkonstruktivistischer Perspektive Schmidt 1994 und Keppler 2005.

25 Alternative Sichtweisen liefern Röhle (2010: passim) zu Google und Pscheida (2010: 331-447) zu Wikipedia. 
Dieses empirisch zu klärende Problem verweist auf die generelle Frage nach den Auswirkungen neuartiger Prozesse der Verbreitung und Legitimierung von Wissen bzw. nach einem gänzlich neuen Modus der (medialen) Konstruktion von Wirklichkeit (vgl. hierzu die Beiträge in Wolling et al. 2010). In Bezug auf die von mir postulierten Schattenzonen der Wissensordnung könnte jener netzwerkmediale Modus der Distribution von Wissen dazu führen, dass sowohl kulturelle Tabus ihre Wirksamkeit als auch abweichend-klandestine Deutungsmuster ihren spezifischen Status verlieren - etwa weil die Möglichkeit weitgehend anonymer Kommunikation $^{26}$ die individuelle und soziale Relevanz der Bewahrung reflexiver Geheimnisse verringert. Wenn wir dieser These folgten, ermöglichten die Netzwerkmedien das ,Gespräch' über alle Themen, so dass der Raum des kulturell Unsagbaren letztlich immer kleiner würde. Dies könnte zumindest für jene Wissensbestände gelten, die aufgrund moralisch-normativen Drucks der Gesamtkultur bislang nicht prozessierbar waren, obwohl die Träger des betreffenden Wissens es gern geteilt hätten. Anders sieht die Situation hingegen bei jenem Wissen aus, das aus machtstrategischen oder sonstigen Gründen verborgen bleiben soll und bei dem es gar kein Bedürfnis der Überführung des kryptodoxen in heterodoxes oder gar orthodoxes Wissen gibt. In diesem Falle stellten die Netzwerkmedien über mehr oder weniger hermetisch abgeschlossene Foren gänzlich neue, geschützte Diskursräume bereit, in denen kryptodoxes Wissen unter ,Eingeweihten' (die sich nicht einmal persönlich kennen müssen) sehr viel einfacher ausgetauscht werden könnte als früher.

Es mag also durchaus sein, dass kryptodoxes Wissen tendenziell eher in der Vergangenheit anzutreffen ist. Es kann aber auch sein, dass sich in Folge der Veränderungen beim Umgang mit Wissen in unserer Gesellschaft lediglich die Art und Weise wandelt, in der Schattenwelten entstehen, sich stabilisieren oder eben hier und da auch aufgedeckt und dadurch als solche zerstört werden. Ich denke, das Letztere ist der Fall. Und lediglich der notwendig historische Charakter der bereits aufgedeckten Schattenzonen lässt den Eindruck entstehen, dass das Phänomen als solches eher der Vergangenheit angehört. Zu klären sind diese und andere Fragen jedoch nur durch ein empirisches Forschungsprogramm zur Wissenssoziologie kryptodoxen Wissens, dessen Grundzüge erst noch formuliert werden müssen.

\section{Literatur}

Anton, Andreas (2011): Unwirkliche Wirklichkeiten. Zur Wissenssoziologie von Verschwörungstheorien, Berlin.

Baroja, Julio Caro (1967): Die Hexen und ihre Welt, Stuttgart (span. Orig. 1961).

Baudrillard, Jean (1978): Agonie des Realen, Berlin.

Beck, Birgit (1995): Vergewaltigung von Frauen als Kriegsstrategie im Zweiten Weltkrieg?, in: Andreas Gestrich (Hrsg.), Gewalt im Krieg. Ausübung, Erfahrung und Verweigerung von Gewalt in den Kriegen des 20. Jahrhunderts, Münster, S. 34-50.

Bellebaum, Alfred (1992): Schweigen und Verschweigen. Bedeutungen und Erscheinungsvielfalt einer Kommunikationsform, Opladen.

Berger, Peter L. / Thomas Luckmann (1991): Die gesellschaftliche Konstruktion der Wirklichkeit. Eine Theorie der Wissenssoziologie, Frankfurt / Main (28.-29. Tausend; engl. Original 1966).

Böhle, Fritz (2003): Wissenschaft und Erfahrungswissen - Erscheinungsformen, Voraussetzungen und Folgen einer Pluralisierung des Wissens, in: Stefan Böschen / Ingo Schulz-Schaeffer (Hrsg.), Wissenschaft in der Wissensgesellschaft, Wiesbaden, S. 143-177.

Bornemann, Ernest (1980 a): Die Umwelt des Kindes im Spiegel seiner, verbotenen' Lieder, Reime, Verse und Rätsel, Frankfurt / Main.

26 Wobei die Idee einer vollständigen Anonymität von Sprechern in der Netzwerkkommunikation wohl eher Mythos als Realität darstellt (vgl. Brunst 2009). 
Bornemann, Ernest (1980 b): Unsere Kinder im Spiegel ihrer Lieder, Reime, Verse und Rätsel, Frankfurt / Main.

Bornemann, Ernest (1981): Die Welt der Erwachsenen in den ,verbotenen' Reimen deutschsprachiger Stadtkinder, Frankfurt / Main.

Bourdieu, Pierre (1993): Über einige Eigenschaften von Feldern, in: Ders., Soziologische Fragen, Frankfurt / Main, S. 107-114.

Brunst, Phillip (2009): Anonymität im Internet - rechtliche und tatsächliche Rahmenbedingungen, Berlin.

Connerton, Paul (2008): How Modernity Forgets, Cambridge.

Davies, Paul C.W. et al. (2009): Signatures of a Shadow Biosphere, in: Astrobiology 9, S. 241-249.

Degele, Nina (2000): Informiertes Wissen. Eine Wissenssoziologie der computerisierten Gesellschaft, Frankfurt / Main.

Dimbath, Oliver / Peter Wehling (2011 a): Soziologie des Vergessens. Theoretische Zugänge und empirische Forschungsfelder, Konstanz.

Dimbath, Oliver / Peter Wehling (2011 b): Soziologie des Vergessens: Konturen, Themen und Perspektiven, in: Dies. (Hrsg.), Soziologie des Vergessens. Theoretische Zugänge und empirische Forschungsfelder, Konstanz, S. 7-34.

Doering-Manteuffel, Sabine (2008): Das Okkulte. Eine Erfolgsgeschichte im Schatten der Aufklärung. Von Gutenberg bis zum World Wide Web, München.

Duerr, Hans Peter (Hrsg.)(1981): Der Wissenschaftler und das Irrationale. Erster Band: Beiträge aus Ethnologie und Anthropologie, Frankfurt / Main.

Eid, Volker (2002): Alle wissen alles, aber keiner weiß Bescheid. Über Wissens-Optimismus und frustrierende Unübersichtlichkeit, in: Reinhardt Rüdel / Carmen Städelhofer (Hrsg), Wohin geht die Wissensgesellschaft, Bielefeld, S. 129-145.

Enzensberger, Hans Magnus (Hrsg.) (2003): Anonyma. Eine Frau in Berlin. Tagebuchaufzeichnungen vom 20. April bis 22. Juni 1945, Frankfurt / Main.

Esposito, Elena (2002): Soziales Vergessen. Formen und Medien des Gedächtnisses der Gesellschaft, Frankfurt / Main.

Esser, Hartmut (1996): Die Definition der Situation, in: Kölner Zeitschrift für Soziologie und Sozialpsychologie 48, S. 1-34.

Favret-Saada, Jeanne (1979): Die Wörter, der Zauber, der Tod. Der Hexenglaube im Hainland von Westfrankreich, Frankfurt / Main (franz. Orig. 1977).

Friederichs, Günther (2001): Mode 2 und Erkenntnis, in: Gerd Bender (Hrsg.), Neue Formen der Wissenserzeugung, Frankfurt / Main, S. 69-82.

Foucault, Michel (1996): Wahnsinn und Gesellschaft. Eine Geschichte des Wahns im Zeitalter der Vernunft, Frankfurt / Main (franz. Orig. 1961).

Foucault, Michel (1991): Die Ordnung des Diskurses, Frankfurt / Main (franz. Orig. 1972).

Freud, Sigmund (2007): Totem und Tabu. Einige Übereinstimmungen im Seelenleben der Wilden und der Neurotiker, Frankfurt / Main (zehnte, unveränderte Auflage; Orig. 1913).

Fuchs-Heinritz, Werner / Alexandra König (2005): Pierre Bourdieu. Eine Einführung, Konstanz.

Goffman, Erving (1991): Wir alle spielen Theater. Die Selbstdarstellung im Alltag, München.

Hauschild, Thomas (2002): Magie und Macht in Italien. Über Frauenzauber, Kirche und Politik, Gifkendorf.

Hultkrantz, Åke (1981): Ritual und Geheimnis: Über die Kunst der Medizinmänner, oder: Was der Herr Professor verschwieg, in: Hans Peter Duerr (Hrsg.), Der Wissenschaftler und das Irrationale. Erster Band: Beiträge aus Ethnologie und Anthropologie, Frankfurt / Main, S. 73-97. 
Keppler, Angela (2005): Medien und soziale Wirklichkeit, in: Michael Jäckel (Hrsg.), Mediensoziologie. Grundfragen und Forschungsfelder, Wiesbaden, S. 91-106.

Klein, Alexandra (2010): Jugend, Medien und Pornographie, in: Michael Schetsche / Renate-Berenike Schmidt (Hrsg.), Sexuelle Verwahrlosung. Empirische Befunde - Gesellschaftliche Diskurse - Sozialethische Reflexionen, Wiesbaden, S. 167-183.

Knoblauch, Hubert (2005): Wissenssoziologie, Konstanz.

Kuwert, Phillip et al. (2010): Trauma and Current Posttraumatic Stress Symptoms in Elderly German Women Who Experienced Wartime Rapes in 1945, in: The Journal of Nervous and Mental Disease 198, S. $450-451$.

Lakoff, George / Johnson, Mark (1980): Metaphors We Live By. Chicago.

Luhmann, Niklas (1996): Die Realität der Massenmedien, Opladen (2., erw. Aufl.).

Marton, Yves (2002): „I saw it with my own eyes“. An ethnography of visions and other anomalous phenomena among participants in Candomble, Umbanda and spiritism from Rio de Janeiro and Abadiania, Goias, Los Angeles: University of California.

Plaß, Christine / Michael Schetsche (2001): Grundzüge einer wissenssoziologischen Theorie sozialer Deutungsmuster, in: Sozialer Sinn 3, S. 511-536.

Pscheida, Daniela (2010): Das Wikipedia-Universum. Wie das Internet unsere Wissenskultur verändert, Bielefeld.

Richard, Birgit (2010): Sexualisierte Jugendliche Netzkulturen?, in: Michael Schetsche / Renate-Berenike Schmidt (Hrsg.), Sexuelle Verwahrlosung. Empirische Befunde - Gesellschaftliche Diskurse - Sozialethische Reflexionen, Wiesbaden, S. 185-205.

Röhle, Theo (2010): Der Google-Komplex. Über Macht im Zeitalter des Internet, Bielefeld.

Roitzsch, Peter (2008): Das Voynich-Manuskript - Ein ungelöstes Rätsel der Vergangenheit, Münster.

Rudolph, Ebermut (1977): Die geheimnisvollen Ärzte: von Gesundbetern und Spruchheilern, Freiburg.

Sander, Helke / Barbara Johr (2005): BeFreier und Befreite. Krieg, Vergewaltigung, Kinder, Frankfurt / Main.

Schetsche, Michael (2008): Das Geheimnis als Wissensform. Soziologische Anmerkungen, in: Journal for Intelligence, Propaganda and Security Studies 2, S. 33-50.

Schetsche, Michael (2010): Das Internet, das sexuelle Geheimnis und das Ende der Pornografie, in: Thorsten Benkel / Fehmi Akalin (Hrsg.), Soziale Dimensionen der Sexualität, Gießen, S. 319-337.

Schetsche, Michael / Renate-Berenike Schmidt (Hrsg.) (2010): Sexuelle Verwahrlosung. Empirische Befunde - Gesellschaftliche Diskurse - Sozialethische Reflexionen, Wiesbaden.

Schirrmeister, Claudia (2004): Geheimnisse. Über die Ambivalenz von Wissen und Nicht-Wissen, Wiesbaden.

Schmidt, Renate-Berenike / Michael Schetsche (1998): Jugendsexualität und Schulalltag, Opladen.

Schmidt, Renate-Berenike / Michael Schetsche (2009): Sexuelle Sozialisation. Sechs Annäherungen, Berlin.

Schmidt, Siegfried J. (1994): Die Wirklichkeit des Beobachters, in: Klaus Merten et al. (Hrsg.), Die Wirklichkeit der Medien. Eine Einführung in die Kommunikationswissenschaft, Opladen, S. 3-19.

Schmidt-Lux, Thomas (2008): Wissenschaft als Religion. Szientismus im ostdeutschen Säkularisierungsprozess, Würzburg.

Sievers, Burkard (1974): Geheimnis und Geheimhaltung in sozialen Systemen, Opladen.

Simmel, Georg (1907): Das Geheimnis. Eine sozialpsychologische Skizze, in: Der Tag, 10.12.1907, abrufbar unter http://socio.ch/sim/verschiedenes/1907/geheimnis.htm, letztes Abrufdatum: 3.2.2012

Simmel, Georg (1958): Soziologie. Untersuchung über die Formen der Vergesellschaftung, Berlin.

Sorell, Tom (1991): Scientism. Philosophy and the Infatuation with Science, London. 
von Münch, Ingo (2009): „Frau, komm!“ Die Massenvergewaltigung deutscher Frauen und Mädchen 1944/45, Graz.

Weis, Kurt (2009): Wege zum Erfahrungswissen. Ein Zwischenbericht aus dem Urwald, in: Eva-Maria Glasbrenner / Christian Hackbarth-Johnson (Hrsg.), Einheit der Wirklichkeiten. Festschrift für Michael von Brück, München, S. 77-98.

Westerbarkey, Joachim (1991): Das Geheimnis. Zur funktionalen Ambivalenz von Kommunikationsstrukturen, Opladen.

Wolling, Jens / Andreas Will / Christina Schumann (Hrsg.) (2010): Medieninnovationen. Wie Medien die Kommunikation in der Gesellschaft verändern, Konstanz.

Young, David E. / Jean-Guy Goulet (Hrsg.) (1994): Being Changed. The Anthropology of Extraordinary Experience, Peterborough.

PD Dr. Michael Schetsche Albert-Ludwigs-Universität Freiburg Institut für Soziologie Rempartstr. 15 79085 Freiburg michael.schetsche@soziologie.uni-freiburg.de 